\title{
Psychologische Merkmale jugendlicher Patienten vor einer
}

\section{Gewichtsreduktionsbehandlung}

\section{Psychological parameters of adolescent patients prior to obesity treatment}

Julius L. Breinker ${ }^{1}$, Ricarda Schmidt ${ }^{1}$, Claudia Hübner ${ }^{1}$, Jana Cämmerer ${ }^{2}$, Antje Körner ${ }^{1}$, Elena Sergeyev ${ }^{1}$, Wieland Kiess ${ }^{2,3} \&$ Anja Hilbert $^{1}$

- Originalarbeit -

- Akzeptierte Manuskriptversion -

\section{Affiliation:}

${ }^{1}$ Universitätsmedizin Leipzig, Integriertes Forschungs- und Behandlungszentrum (IFB)

AdipositasErkrankungen, Forschungsbereich Verhaltensmedizin, Klinik und Poliklinik für Psychosomatische Medizin und Psychotherapie, Leipzig, Deutschland

${ }^{2}$ Universitätsmedizin Leipzig, Klinik und Poliklinik für Kinder- und Jugendmedizin, Zentrum für Pädiatrische Forschung, Leipzig, Deutschland

${ }^{3}$ Universität Leipzig, LIFE Forschungszentrum für Zivilisationskrankheiten, Leipzig, Deutschland

\section{Korrespondenzadresse:}

Julius L. Breinker, Universitätsmedizin Leipzig, Integriertes Forschungs- und Behandlungszentrum (IFB) AdipositasErkrankungen, Bereich Verhaltensmedizin, Klinik und Poliklinik für Psychosomatische Medizin und Psychotherapie, Philipp-Rosenthal-Straße 27, 04103 Leipzig

E-Mail: julius.breinker@medizin.uni-leipzig.de 


\section{Schlüsselwörter:}

Adipositas, Übergewicht, Jugendliche, psychologisch, Psychopathologie

Obesity, overweight, adolescents, psychological, psychopathology

Die veröffentlichte Verlagsversion ist verfügbar unter / The final publication is available at:

https://www.thieme-connect.de/products/ejournals/abstract/10.1055/a-1197-3155

(c) 2020. Thieme. All rights reserved. 
Psychologische Merkmale jugendlicher Patienten vor einer Gewichtsreduktionsbehandlung

\section{Zusammenfassung}

Übergewicht und Adipositas zeigen bei Kindern und Jugendlichen eine hohe

Prävalenz und Persistenz. Entsprechende Therapien sind kaum individualisiert und zeigen nur moderate Erfolge. Die Psychopathologie bleibt zumeist unberücksichtigt. Die vorliegende Studie hat die Psychopathologie von Jugendlichen mit Übergewicht und Adipositas $(N=201$, Alter 12 - 17 Jahre) vor einer Gewichtsreduktionsbehandlung erhoben und Alters- sowie

Geschlechtseffekte untersucht. Mittels Fragebögen wurden selbstberichtet die allgemeine Symptombelastung, Essstörungs- und allgemeine Psychopathologie, gewichtsbezogene Selbststigmatisierung und die körperliche und psychische Lebensqualität erhoben. Mädchen zeigten hypothesenkonform ein höheres Maß an gewichtsbezogener Selbststigmatisierung und eine stärkere Essstörungspsychopathologie als Jungen. Ältere Jugendliche berichteten eine geringere Lebensqualität als jüngere. Vergleiche zu Referenzgruppen aus Bevölkerungs- und Normierungsstichproben zeigten auffälligere Werte bei Jugendlichen mit Übergewicht und Adipositas für alle untersuchten Parameter. Eine auffällige Psychopathologie ist demzufolge ein wichtiges Korrelat bei Jugendlichen mit hohem Gewichtsstatus und ein potenzieller Einflussfaktor auf den Erfolg einer Gewichtsreduktionsbehandlung. Zukünftige Studien sollten die Psychopathologie bei Übergewicht und Adipositas weiter differenzieren und therapeutische Ressourcen evaluieren.

\footnotetext{
Abstract

Overweight and obesity in children and adolescents are highly prevalent and persistent. Current weight loss treatments are rarely individualized and show only small to moderate efficacy. Only rarely, psychopathological parameters are considered. The present study evaluated the psychopathology of adolescents with overweight and obesity $(N=201$, ages $12-17$ years) prior to obesity treatment. The data were analyzed for age and sex effects. Self-report questionnaires assessed general symptom burden, eating disorder and general
} 
Psychologische Merkmale jugendlicher Patienten vor einer Gewichtsreduktionsbehandlung

psychopathology, weight-related self-stigmatization, and physical and mental quality of life. Girls showed higher rates of weight-related self-stigmatization and higher disordered eating behavior compared to boys. Older adolescents reported a lower quality of life compared to younger adolescents. In normative comparisons with population-based samples and norms, adolescents with overweight and obesity showed significantly adverse outcomes in all parameters. Thus, this study identified psychopathology as an important factor in adolescents with high weight status that may affect obesity treatment. Future studies should examine psychopathology more differentially and determine therapeutic resources in adolescent overweight and obesity.

Einleitung

Trotz stagnierender Prävalenzraten in den letzten Jahren [1] liegt die Prävalenz für Übergewicht und Adipositas bei 3- bis 17-Jährigen in Deutschland bei $9.5 \%$ bzw. $5.9 \%$ [1]. Die Bedeutsamkeit einer wirksamen Behandlung von Übergewicht und Adipositas im Jugendalter ergibt sich durch ihre Persistenz [2] sowie eine Vielzahl psychischer und physischer Komorbiditäten [3-17]. Als Therapie der Wahl bei Adipositas im Kindes- und Jugendalter gilt ein multimodales Behandlungskonzept mit verhaltens- und familientherapeutischen Aspekten [18], dessen Effekte jedoch insgesamt als niedrig bis moderat einzuschätzen sind [19]. Die Psychopathologie der Teilnehmer bleibt hierbei meist unberücksichtigt. Jedoch zeigte beispielsweise eine australische Studie mit $N=250$ Kindern und Jugendlichen mit Adipositas negative Effekte durch soziale und Verhaltensprobleme sowie positive Effekte durch ein hohes Selbstwertgefühl auf die Gewichtsreduktion [20]. Psychopathologische Aspekte sind wichtige ätiologische Merkmale von Übergewicht und Adipositas im Kindes- und Jugendalter [3]. Internationale Evidenz indiziert bei Jugendlichen mit Übergewicht bzw. Adipositas Auffälligkeiten vor allem im Bereich der Essstörungspsychopathologie [3, 4], gewichtsbezogenen Selbststigmatisierung [5], einer 
Psychologische Merkmale jugendlicher Patienten vor einer Gewichtsreduktionsbehandlung

reduzierten Lebensqualität [3, 4, 6-8], allgemeinen Psychopathologie [9], Problemen mit Gleichaltrigen [9], affektiven Störungen [3, 4], und geringem Selbstwert [3, 4] im Vergleich zu Jugendlichen mit Normalgewicht. Im deutschsprachigen Raum ist die Befundlage hinsichtlich der Psychopathologie bei juveniler Adipositas jedoch gering. Eine deutsche Studie mit $N=41$ behandlungsaufsuchenden Jugendlichen mit Übergewicht bzw. Adipositas fand eine erhöhte Essstörungspsychopathologie sowie eine niedrigere Lebensqualität im Vergleich zu Normwerten [10]. Auch in der deutschen Allgemeinbevölkerung konnte eine niedrigere Lebensqualität bei $N=6813$ 11- bis 17-Jährigen mit Übergewicht bzw. Adipositas im Vergleich zu Jugendlichen mit Normalgewicht beobachtet werden [11]. Eine geringere subjektive Gesundheit, geringeres emotionales Wohlbefinden und weniger Kontakt zu Freunden im Vergleich zu einer schulbasierten Stichprobe demonstrierte eine Studie mit $N=$ 125 behandlungsaufsuchenden Kindern und Jugendlichen zwischen 6 und 16 Jahren mit Übergewicht bzw. Adipositas im Selbstbericht [12]. Betreffend des körperlichen und psychischen Wohlbefindens berichteten $N=1916$ behandlungsaufsuchende 8- bis 17-Jährige aus Deutschland mit Übergewicht bzw. Adipositas auffälligere Werte im Vergleich zu einer Normstichprobe [13]. Zwar fand sich bei $N=191$ behandlungsaufsuchenden Jugendlichen (Alter 13 bis 19 Jahre) mit Übergewicht bzw. Adipositas vor Behandlungsbeginn eine positive Assoziation zwischen dem Body Mass Index $\left(\mathrm{BMI}, \mathrm{kg} / \mathrm{m}^{2}\right)$ und einer gewichtsbezogenen Selbststigmatisierung, Vergleiche mit Kontrollgruppen blieben jedoch aus [14]. Obgleich bevölkerungsbasierte Studien aus Deutschland Alters- und Geschlechtseffekte der Psychopathologie im Jugendalter zeigen (siehe Tabelle 1 sowie [21, 22]), ist der Einfluss von Alter und Geschlecht auf die Psychopathologie im Rahmen juveniler Adipositas kaum untersucht, sowohl in internationalen als auch nationalen Studien [3]. So indizieren internationale Studien bei Kindern und Jugendlichen mit Übergewicht bzw. Adipositas auffälligere Befunde bei Mädchen für Essstörungspsychopathologie [4, 15-17], Körperunzufriedenheit [4], Selbstbewusstsein [4] und Lebensqualität [4, 8] im Vergleich zu 
Psychologische Merkmale jugendlicher Patienten vor einer Gewichtsreduktionsbehandlung

Jungen, sowie bei beiden Geschlechtern eine mit dem Alter zunehmende

Essstörungspsychopathologie [17]. Die wenigen Befunde deutscher Studien zeigten für behandlungsaufsuchende Stichproben mit juveniler Adipositas eine größere gewichtsbezogene Selbststigmatisierung bei Mädchen gegenüber Jungen [14], eine niedrigere Lebensqualität bei jugendlichen Mädchen gegenüber Jungen [13] sowie eine Abnahme der Lebensqualität mit dem Alter bei Mädchen [13].

Zusammenfassend indizieren die wenig verfügbaren Studien psychopathologische Auffälligkeiten bei Jugendlichen mit Übergewicht und Adipositas, die gleichzeitig einen Einfluss auf den Therapieerfolg haben können [20]. Alters- und Geschlechtseffekte wie sie bei Kindern und Jugendlichen der Allgemeinbevölkerung zu finden sind, wurden bei juveniler Adipositas nur selektiv untersucht, vor allem für Deutschland, und ohne Bezug zu entsprechenden Referenzwerten. Ziel der vorliegenden Studie war es daher, psychopathologische Merkmale bei Jugendlichen mit Übergewicht und Adipositas vor Beginn einer multimodalen Adipositastherapie in Abhängigkeit demografischer Charakteristika zu untersuchen. Diese Subgruppenanalysen ermöglichen gleichzeitig die Identifizierung von psychologischen Risikogruppen für den Behandlungskontext. Angenommen wurde, dass hinsichtlich der untersuchten psychopathologischen Faktoren (allgemeine körperliche und psychische Beschwerdelast, allgemeine und Essstörungspsychopathologie, gewichtsbezogene Selbststigmatisierung, gesundheitsbezogene Lebensqualität) Mädchen ungünstigere Werte als Jungen aufweisen und die psychologischen Beeinträchtigungen geschlechtsunabhängig mit dem Alter zunehmen.

Methode

Teilnehmer

Eingeschlossen wurden Jugendliche zwischen 12 und 17 Jahren mit Übergewicht und Adipositas (BMI-Perzentil > 90 bzw. > 97 [18]), die am Integrierten Forschungs- und 
Psychologische Merkmale jugendlicher Patienten vor einer Gewichtsreduktionsbehandlung

105 Behandlungszentrum (IFB) AdipositasErkrankungen in der AdipositasAmbulanz der Klinik

106 und Poliklinik für Kinder- und Jugendmedizin an der Universitätsmedizin Leipzig eine

107 Gewichtsreduktionsbehandlung aufsuchten. Bei Erstvorstellung der Patienten wurde neben

108 einer allgemeinen medizinischen Untersuchung das psychopathologische Profil mit Hilfe von

109 Fragebögen erhoben. Zuvor wurde per Informed Consent das schriftliche Einverständnis zur

110 Verwendung der Daten im Rahmen von Studien eingeholt. Die Studie wurde von der

111 Ethikkommission an der Medizinischen Fakultät der Universität Leipzig genehmigt. Der

112 Zeitraum der Datenerhebung umfasste Juni 2015 bis Oktober 2019.

Messverfahren

Health Behavior in School Children - Symptom Checklist (HBSC-SCL). Mittels acht

Items erfasst der Fragebogen allgemeine Beschwerden wie Kopfschmerz, Bauchschmerz oder

Rückenschmerz der letzten sechs Monate auf einer 5-stufigen Skala $(0=$ „fast täglich“ bis $4=$

(Cronbach's $\alpha=.81$ in der vorliegenden Studie).

Child Eating Disorder Examination-Questionnaire 8 (ChEDE-Q8). In der Kurzform mit acht Items misst der Fragebogen die allgemeine Essstörungspsychopathologie der letzten 28 Tage auf einer 7-stufigen Skala $(0=$ Merkmal war nicht vorhanden bis $6=$ Merkmal war jeden Tag bzw. in extremer Ausprägung vorhanden) [24]. Ein höherer Gesamt-Mittelwert

124 zeigt eine stärkere Essstörungspsychopathologie an (Cronbach’s $\alpha=.80$ ).

Weight Bias Internalization Scale (WBIS). Der Fragebogen erfasst die gewichtsbezogene Selbststigmatisierung und umfasst 11 Items auf einer 7-stufigen Skala $(1=$ „stimme überhaupt nicht zu“ bis 7 = ,stimme voll und ganz zu“) [25]. Ein höherer GesamtMittelwert weist auf eine stärkere Selbststigmatisierung hin (Cronbach's $\alpha=.73$ ). 
Psychologische Merkmale jugendlicher Patienten vor einer Gewichtsreduktionsbehandlung

131 den Subskalen Körper und Psyche mit je vier Items, die mithilfe einer 5-stufigen Skala (1 =

132 „nie“ bis $5=$,immer“) mit Bezug auf die letzte Woche beantwortet werden. Nach

133 Werttransformationen liegen die Skalenwerte zwischen 0 und 100, wobei höhere Werte eine

134 bessere Lebensqualität anzeigen [26] (KINDL-R Körper: Cronbach's $\alpha=.70$ und KINDL-R

135 Psyche: $\alpha=.73$ ).

136 Strengths and Difficulties Questionnaire $(S D Q)$. Der Fragebogen ermittelt juvenile

137 Verhaltensaspekte in Form von Stärken und Schwächen auf den fünf Subskalen emotionale

138 Probleme, Verhaltensprobleme, Hyperaktivität, Verhaltensprobleme mit Gleichaltrigen und

139 prosoziales Verhalten [27]. Er besteht aus 25 Items, die mithilfe einer 3-stufigen Skala $(0=$

140 „nicht zutreffend“ bis 2 = „eindeutig zutreffend“) eingeschätzt werden. Aus den

141 Summenwerten der vier Problem-Subskalen (ohne prosoziales Verhalten) wird ein

142 Gesamtwert berechnet. Höhere Werte indizieren mehr Probleme bzw. mehr prosoziales

143 Verhalten. Auf Grund unzureichender interner Konsistenzen der Subskalen wurde lediglich

144 der Gesamtwert genutzt (Cronbach's $\alpha=.81$ ).

145 Größe und Gewicht wurden im Rahmen einer körperlichen Untersuchung objektiv

146 gemessen. Die Berechnung des BMI-Perzentils basierte auf der Referenzpopulation von

147 Jungen und Mädchen im Alter von 0 bis 18 Jahren von Kromeyer-Hauschild et al. [28].

Datenanalyse

Zur Untersuchung von Gruppenunterschieden wurde eine zweifaktorielle multivariate

151 Varianzanalyse (MANOVA) mit den Faktoren Geschlecht (weiblich, männlich) und

152 Altersgruppe (frühe Adoleszenz: 12 bis 14 Jahre, mittlere Adoleszenz: 15 bis 17 Jahre [29])

153 durchgeführt. Als abhängige Variablen wurden die psychopathologischen Fragebögen

154 (HBSC-SCL, ChEDE-Q8, WBIS, KINDL-R Körper, KINDL-R Psyche und SDQ

155 Gesamtwert) verwendet. Für univariate Vergleiche bei signifikanter MANOVA wurde das

156 partielle $\eta^{2}$ als Effektstärke berechnet und gemäß Cohen [30] interpretiert ( $\eta_{\mathrm{p}}{ }^{2} ;$ klein: .01; 
Psychologische Merkmale jugendlicher Patienten vor einer Gewichtsreduktionsbehandlung

157 mittel: .06; groß: .14). Eine Poweranalyse ergab eine Power von $1-\beta=.99$ für die

158 Identifizierung von Haupteffekten mittlerer Effektstärke $\left(f^{2}=.15\right)$.

159

Für die Vergleiche mit Referenzpopulationen aus Bevölkerungs- und

160 Normierungsstichproben (siehe Tabelle 1) wurden $t$-Tests für die psychopathologischen

161 Fragebögen berechnet und die Effektstärke Cohen's $d$ bestimmt (klein: .20; mittel: .50; groß:

$162.80)[30]$. 
Psychologische Merkmale jugendlicher Patienten vor einer Gewichtsreduktionsbehandlung

163

164

Tabelle 1

Referenzgruppen der genutzten Messverfahren

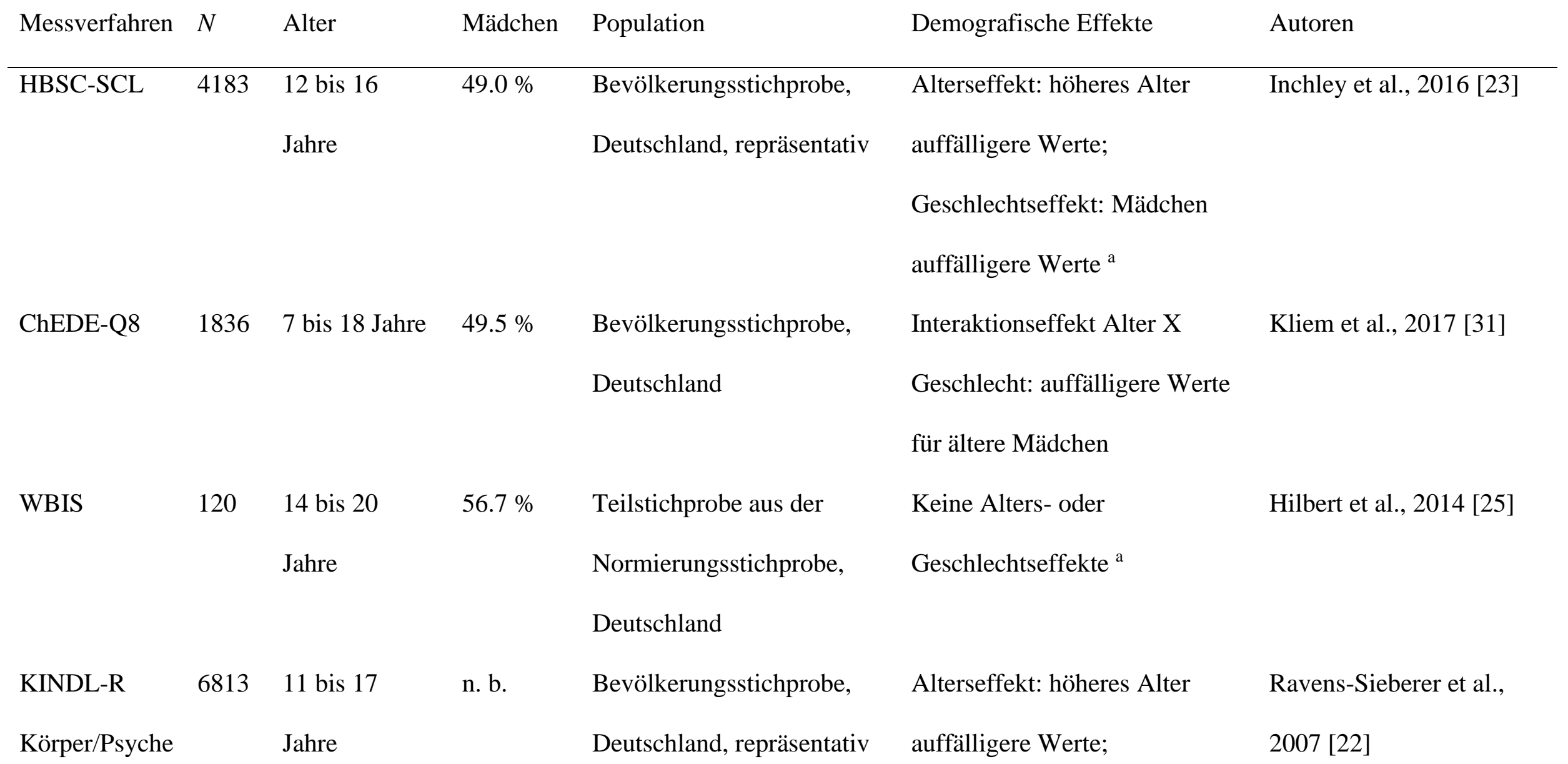


Psychologische Merkmale jugendlicher Patienten vor einer Gewichtsreduktionsbehandlung

Geschlechtseffekt: Mädchen

auffälligere Werte

SDQ

$6726 \quad 11$ bis 17

$48.9 \%$

Bevölkerungsstichprobe,

Deutschland, repräsentativ

Kein Alterseffekt;

Becker et al., 2018 [32]

Jahre

\section{Geschlechtseffekt: Mädchen}

auffälligere Werte
165

166

Anmerkungen. HBSC-SCL = Health Behavior in School Children - Symptom Checklist; ChEDE-Q8 = Child Eating Disorder Examination-

Questionnaire 8; WBIS = Weight Bias Internalization Scale; KINDL-R = Fragebogen zur Erfassung der gesundheitsbezogenen Lebensqualität bei

Kindern und Jugendlichen - Revidierte Form; n. b. = nicht berichtet; SDQ = Strengths and Difficulties Questionnaire.

${ }^{\mathrm{a}}=$ Originaldaten 
Psychologische Merkmale jugendlicher Patienten vor einer Gewichtsreduktionsbehandlung

170 Windows durchgeführt wurden, wurde ein zweiseitiges $\alpha$ von .05 zugrunde gelegt.

171

172

173 Teilnehmer

174

175

176

177

178

179

180
Ergebnisse

Wie in Tabelle 2 dargestellt, umfasste die Stichprobe $N=201$ Teilnehmer $(13.81 \pm$ 1.56 Jahre, 54\% weiblich) mit einem mittleren BMI-Perzentil von 98.74 ( $S D=1.51)$. Gemäß BMI-Perzentil lag bei $n=26$ Jugendlichen Übergewicht und bei $n=175$ Jugendlichen Adipositas vor. Die Mädchen waren signifikant älter als die Jungen, $t(199)=2.16, p=.03$.

Bezogen auf den Gewichtsstatus fanden sich weder signifikante Alters- noch

Geschlechterunterschiede zwischen den Gruppen, $t(199)=-0.42, p=.68$ und $\chi^{2}(1, N=201)=$ $.79, p=.38$ 
Psychologische Merkmale jugendlicher Patienten vor einer Gewichtsreduktionsbehandlung

181

Tabelle 2

Deskriptive und Teststatistik für Gruppenunterschiede in demografischen und psychopathologischen Merkmalen $(N=201)$

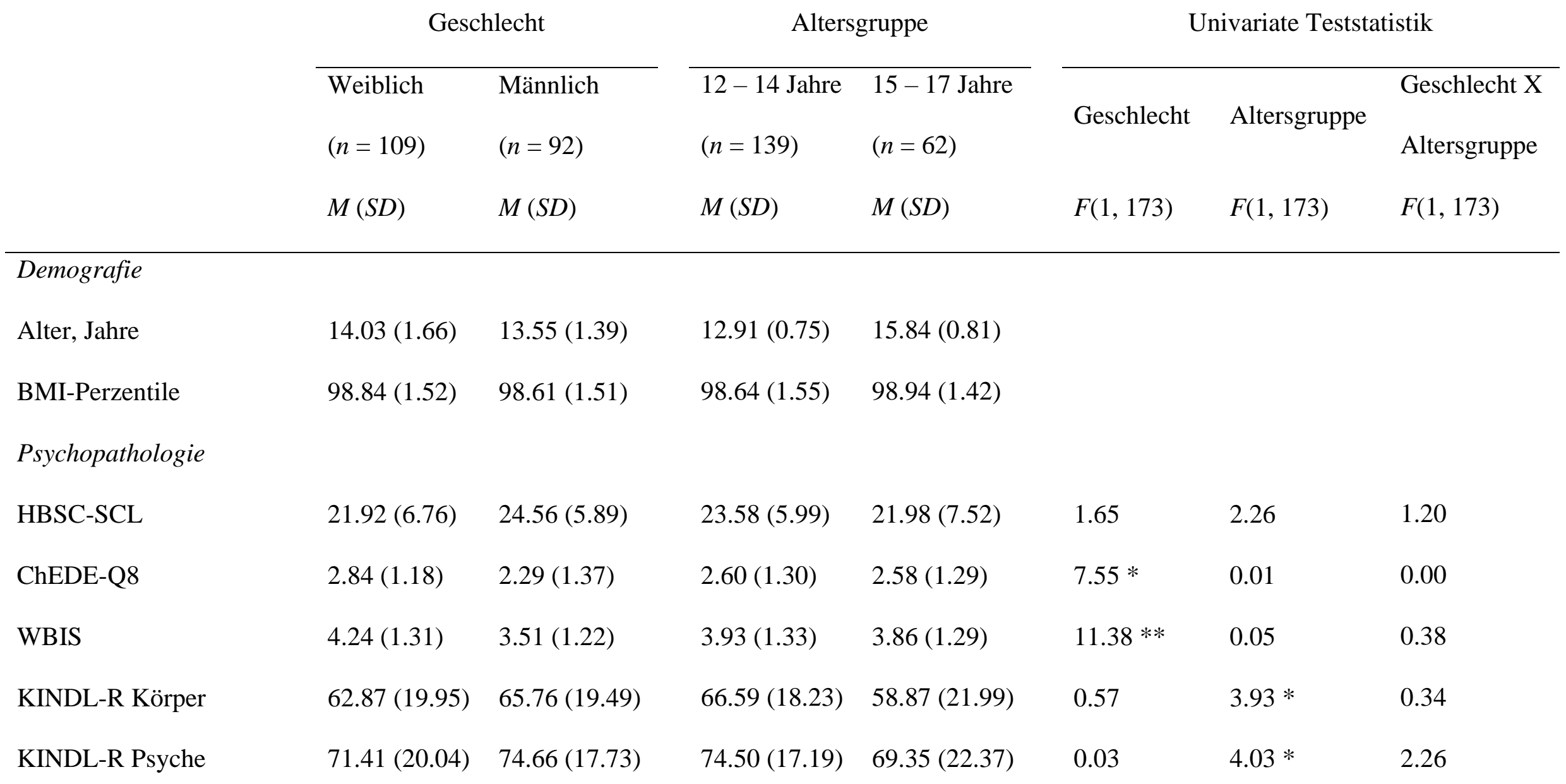


Psychologische Merkmale jugendlicher Patienten vor einer Gewichtsreduktionsbehandlung
SDQ Gesamtwert
$13.34(5.85)$
$12.76(6.59)$
$13.27(6.22)$
$12.63(6.15)$
0.01
0.47
0.00

183

Anmerkungen. BMI = Body-Mass-Index; HBSC-SCL $=$ Health Behavior in School Children - Symptom Checklist; ChEDE-Q8 = Child Eating

184 Disorder Examination-Questionnaire 8; WBIS = Weight Bias Internalization Scale; KINDL-R = Fragebogen zur Erfassung der

185 gesundheitsbezogenen Lebensqualität bei Kindern und Jugendlichen - Revidierte Form; SDQ = Strengths and Difficulties Questionnaire.

$186 *=p<.05, * *=p<.01$. 
Psychologische Merkmale jugendlicher Patienten vor einer Gewichtsreduktionsbehandlung Die MANOVA zeigte signifikante Haupteffekte für das Geschlecht, $F(6,168)=3.02$,

$188 p<.01$, und die Altersgruppe, $F(6,168)=2.38, p=.03$. Die Interaktion Geschlecht $\mathrm{X}$

189 Altersgruppe war nicht signifikant, $F(6,168)=1.45, p=.20$. Für das Geschlecht fanden sich

190 univariat signifikante Unterschiede für die gewichtsbezogene Selbststigmatisierung $(p<.01$,

$\left.191 \eta_{\mathrm{p}}^{2}=.06\right)$ sowie die Essstörungspsychopathologie $\left(p=.01, \eta_{\mathrm{p}}^{2}=.04\right)$. Hierbei wiesen

192 Mädchen eine stärkere gewichtsbezogene Selbststigmatisierung sowie eine auffälligere

193 Essstörungspsychopathologie auf als Jungen. Eine zusätzliche Analyse des Geschlechtseffekts

194 unter Kontrolle des Alters zeigte keine Veränderung in den Ergebnissen. Für die Altersgruppe

195 fanden sich univariat signifikante Unterschiede für die körperliche Lebensqualität $(p=.049$,

$\left.196 \eta_{\mathrm{p}}^{2}=.02\right)$ und die psychische Lebensqualität $\left(p=.046, \eta_{\mathrm{p}}{ }^{2}=.02\right)$. Hierbei zeigten ältere

197 Jugendliche eine niedrige körperliche und psychische Lebensqualität als die jüngeren.

198

Referenzpopulationen

Jugendliche mit Übergewicht bzw. Adipositas zeigten im Vergleich zu den

201 Referenzpopulationen für alle untersuchten psychopathologischen Parameter auffälligere

202 Werte (siehe Tabelle 3). 
Psychologische Merkmale jugendlicher Patienten vor einer Gewichtsreduktionsbehandlung

203 Tabelle 3

204 Vergleich der Stichprobenkennwerte mit denen der Referenzgruppen

\begin{tabular}{lllrrrr}
\hline & Stichprobe & \multicolumn{1}{l}{ Referenz } & & & & \\
& $M(S D)$ & $M(S D)$ & $t$ & $d f$ & $p$ & $d$ \\
\hline HBSC-SCL & $23.10(6.50)$ & $24.05(6.17)$ & -2.03 & 217 & .04 & 0.15 \\
ChEDE-Q8 & $2.59(1.29)$ & $1.05(1.33)$ & 16.03 & 291 & $<.001$ & 1.16 \\
WBIS & $3.90(1.32)$ & $2.67(1.13)$ & 8.85 & 301 & $<.001$ & 0.98 \\
KINDL-R Körper & $64.20(19.74)$ & $70.6(21.06)$ & -4.52 & 213 & $<.001$ & 0.30 \\
KINDL-R Psyche & $72.91(19.04)$ & $81.1(16.85)$ & -6.03 & 209 & $<.001$ & 0.48 \\
SDQ Gesamtwert & $13.07(6.19)$ & $10.0(4.6)$ & 6.97 & 206 & $<.001$ & 0.66
\end{tabular}

205 Anmerkungen. HBSC-SCL = Health Behavior in School Children - Symptom Checklist;

206 ChEDE-Q8 = Child Eating Disorder Examination-Questionnaire 8; WBIS = Weight Bias

207 Internalization Scale; KINDL-R = Fragebogen zur Erfassung der gesundheitsbezogenen

208 Lebensqualität bei Kindern und Jugendlichen - Revidierte Form; SDQ = Strengths and

209 Difficulties Questionnaire. 
Psychologische Merkmale jugendlicher Patienten vor einer Gewichtsreduktionsbehandlung

Diskussion

Die vorliegende Studie untersuchte psychologische Merkmale von Jugendlichen mit

212 Übergewicht und Adipositas vor einer Gewichtsreduktionsbehandlung und deren Assoziation

213 mit demografischen Charakteristika. Hypothesenkonform berichteten Mädchen mit

214 Übergewicht und Adipositas sowie ältere Jugendliche mehr psychologische

215 Beeinträchtigungen als Jungen und jüngere Jugendliche. Im Vergleich zu

216 Referenzstichproben zeigten Jugendliche mit Übergewicht und Adipositas für alle

217 untersuchten psychologischen Parameter auffälligere Werte.

Die Ergebnisse der geschlechtsspezifischen Analysen indizieren innerhalb der Gruppe

219 behandlungsaufsuchender Jugendlicher mit Übergewicht und Adipositas bei Mädchen im

220 Vergleich zu Jungen eine stark erhöhte Essstörungspsychopathologie und gewichtsbezogene

221 Selbststigmatisierung, während sich beide Geschlechter nicht in allgemeiner

222 Psychopathologie und körperlicher Beschwerdelast unterscheiden. Diese

223 geschlechtsspezifische Vulnerabilität für körper- und essensbezogene Psychopathologie stützt

224 bereits gefundene Zusammenhänge bei Jugendlichen mit Übergewicht zwischen dem

225 weiblichen Geschlecht und einer erhöhten gewichtsbezogenen Selbststigmatisierung [14]

226 sowie einer ausgeprägteren Essstörungspsychopathologie [4, 15-17]. Die Ergebnisse

227 bezüglich ausbleibender geschlechtsspezifischer Unterschiede der Lebensqualität

228 widersprechen bisherigen nationalen und internationalen Befunden [4, 8, 13]. Der Schluss

229 liegt nahe, dass die geschlechtsspezifische Einschätzung der Lebensqualität durch den

230 Behandlungsstatus aufgehoben wird und Jungen genauso belastet sind wie Mädchen.

231 Aufgrund fehlender Vorbefunde für allgemeine körperliche und psychische Symptome bei

232 juveniler Adipositas lassen sich Ergebnisse hierzu schwer in Bezug setzen, in der

233 vorliegenden Stichprobe zeigten sich jedoch keine signifikanten Unterschiede zwischen den

234 Geschlechtern und Altersgruppen. 
Psychologische Merkmale jugendlicher Patienten vor einer Gewichtsreduktionsbehandlung

Die gefundenen Alterseffekte für eine geringere körperliche und psychische

236 Lebensqualität der älteren im Vergleich zu den jüngeren Jugendlichen mit Übergewicht bzw.

237 Adipositas stehen im Einklang mit Vorbefunden [8, 13] und könnten auf eine längere

238 Erkrankungsdauer und damit verbundene Symptomexposition zurückgeführt werden.

239 Weiterhin könnten besondere Entwicklungsaufgaben der späten Adoleszenz (soziale

240 Beziehungen, Berufswahl) bei persistierendem Übergewicht bzw. Adipositas in einer

241 geringeren Lebensqualität der älteren Jugendlichen resultieren, während allgemeine

242 körperliche und psychische Belastungsfaktoren im Vergleich zu jüngeren Jugendlichen stabil

243 bleiben. Entgegen der Erwartungen konnte keine zunehmende Psychopathologie mit dem

244 Alter gefunden werden, was darauf schließen lassen könnte, dass psychische Auffälligkeiten

245 bereits ab der frühen Adoleszenz auf einem hohen Niveau stabil bleiben.

246 Die umfassenden Vergleiche psychologischer Faktoren von Jugendlichen mit

247 Übergewicht und Adipositas zu Referenzgruppen bestätigten die Annahme, dass die

248 vorliegende Stichprobe auffälligere Werte bezüglich aller untersuchten Parameter aufweist.

249 Die Unterschiede in körperlichen Symptomen stellen wahrscheinlich direkte gesundheitliche

250 Effekte von Übergewicht bzw. Adipositas dar. Die gefundenen großen Unterschiede in der

251 Essstörungspsychopathologie, Lebensqualität, gewichtsbezogenen Selbststigmatisierung

252 sowie der allgemeinen psychischen Belastung können als psychopathologische Aspekte

253 sowohl ursächlich als auch Folge des Übergewichts bzw. der Adipositas sein [33].

Die in der vorliegenden Stichprobe gefundenen Alters- und Geschlechtseffekte

255 deckten sich nur zum Teil mit den Befunden der Referenzgruppen: Bezüglich

256 gewichtsbezogener Selbststigmatisierung fand sich bei juveniler Adipositas ein

257 Geschlechtseffekt zulasten der Mädchen im Vergleich zu Jungen, gleichwohl fanden sich die

258 Geschlechtseffekte für allgemeine körperliche und psychische Beschwerdelast sowie

259 Lebensqualität der Referenzgruppen nicht bei juveniler Adipositas wieder. Ältere Jugendliche

260 mit Adipositas zeigten übereinstimmend mit der Referenzgruppe eine geringere 
Psychologische Merkmale jugendlicher Patienten vor einer Gewichtsreduktionsbehandlung

261 Lebensqualität als jüngere Jugendliche, während keine Alterseffekte für körperliche

262 Symptome, Essstörungspsychopathologie und gewichtsbezogene Selbststigmatisierung

263 gefunden wurden. Die im Vergleich zu Referenzgruppen erhöhten Auffälligkeiten aller

264 erhobenen Psychopathologien bei juveniler Adipositas könnten Alters- und

265 Geschlechtseffekte aus den Referenzgruppen somit überlagern. Gleichzeitig scheinen

266 Geschlechtseffekte für gewichtsspezifische Parameter wie die gewichtsbezogene

267 Selbststigmatisierung bei juveniler Adipositas noch verstärkt zu werden.

Zu den Stärken der Studie zählt die ausgewogene Verteilung der Stichprobe in Bezug

auf Alter und Geschlecht, sowie die objektive Bestimmung von Übergewicht bzw. Adipositas.

Zudem ließ sich durch verschiedene etablierte Fragebögen ein differenziertes psychologisches

271 Profil der Jugendlichen erheben. Durch die Nutzung standardisierter Fragebogenverfahren

272 wurde eine hohe interne Vergleichbarkeit der Ergebnisse sowie mit Referenzpopulationen

273 erzielt, jedoch wurden keine diagnostischen Interviews geführt, die eine objektivere

274 Einschätzung psychologischer Symptome zuließen als der reine Selbstbericht. Obgleich keine

275 Unterschiede zwischen Jugendlichen mit Übergewicht und Adipositas im psychologischen

276 Profil in der Stichprobe gefunden wurden (Ergebnisse nicht berichtet), könnte der Einschluss

277 von Jugendlichen mit Übergewicht in die Stichprobe aus Jugendlichen mit Adipositas zu einer

278 Unterschätzung der Effekte beigetragen haben und die Generalisierbarkeit der Ergebnisse auf

279 die juvenile Adipositas einschränken. Die genutzten Referenzgruppen wurden in

280 bestmöglicher Übereinstimmung hinsichtlich der vorliegenden Stichprobe sowie Aktualität

281 ausgewählt. Dennoch handelt es sich um verschiedene Populationen, die nicht zeitgleich

282 erhoben wurden. Darüber hinaus lagen nicht für alle Referenzgruppen Originaldatensätze vor,

283 sodass keine direkten Vergleichsanalysen zwischen den Kontrollgruppen und der

284 vorliegenden Stichprobe hinsichtlich Subgruppenunterschieden vorgenommen werden

285 konnten. 
Psychologische Merkmale jugendlicher Patienten vor einer Gewichtsreduktionsbehandlung Zukünftige Studien sollten die Unterschiede zwischen Mädchen und Jungen mit

287 Übergewicht bzw. Adipositas hinsichtlich sozialer Aspekte und psychischer Komorbiditäten weiter differenzieren, um eventuell moderierenden Faktoren in der

Gewichtsreduktionsbehandlung besser begegnen zu können. Weiterhin sollten für gezieltere

Therapiemethoden und bessere Therapieergebnisse psychopathologische Veränderungen

291 durch das Alter bzw. die Entwicklungsaufgaben der Jugendlichen evaluiert und das

292 psychologische Profil um therapeutische Ressourcen ergänzt werden. Schließlich ermöglichen

293 longitudinal angelegte Studien mit einem größeren Altersbereich die Betrachtung

294 intraindividueller Veränderungen im psychopathologischen Profil im Rahmen einer

295 Gewichtsreduktionsbehandlung und deren Vorhersagewert auf den Erfolg dieser [20].

Die vorliegenden Ergebnisse indizieren ein auffälliges psychopathologisches Profil bei Jugendlichen mit Übergewicht und Adipositas vor einer Gewichtsreduktionsbehandlung. Da eine erhöhte Psychopathologie bei Jugendlichen mit Übergewicht und Adipositas Einfluss auf die Behandlung nehmen kann [20], ist es wichtig, diese Auffälligkeiten in der Therapie zu berücksichtigen. Insbesondere bei Mädchen sollte eine mögliche Essstörungspsychopathologie und Selbststigmatisierung beachtet werden. Die geringere körperliche und psychische Lebensqualität der älteren Jugendlichen mit Übergewicht und Adipositas verdeutlicht die Wichtigkeit einer frühzeitigen Intervention. 
Psychologische Merkmale jugendlicher Patienten vor einer Gewichtsreduktionsbehandlung

Die Autoren erklären, dass keine Interessenkonflikte vorliegen.

\section{Literatur}

[1] Schienkiewitz A, Brettschneider A-K, Damerow S, et al. Übergewicht und Adipositas im Kindes- und Jugendalter in Deutschland - Querschnittergebnisse aus KiGGS Welle 2 und Trends. Journal of Health Monitoring 2018; 3: 16-23. doi:10.17886/RKI-GBE2018-005.2

[2] Simmonds M, Llewellyn A, Owen CG, et al. Predicting adult obesity from childhood obesity: a systematic review and meta-analysis. Obes Rev 2016; 17: 95-107. doi:10.1111/obr.12334

[3] Rankin J, Matthews L, Cobley S, et al. Psychological consequences of childhood obesity: psychiatric comorbidity and prevention. Adolesc Health Med Ther 2016; 7: 125-146. doi:10.2147/AHMT.S101631

[4] Gibson LY, Allen KL, Davis E, et al. The psychosocial burden of childhood overweight and obesity: evidence for persisting difficulties in boys and girls. Eur J Pediatr 2017; 176: 925-933. doi:10.1007/s00431-017-2931-y

[5] Puhl RM, Himmelstein MS. Weight bias internalization among adolescents seeking weight loss: Implications for eating behaviors and parental communication. Front Psychol 2018; 9: 2271. doi:10.3389/fpsyg.2018.02271

[6] Anderson YC, Wynter LE, Treves KF, et al. Assessment of health-related quality of life and psychological well-being of children and adolescents with obesity enrolled in a New Zealand community-based intervention programme: an observational study. BMJ Open 2017; 7: e015776. doi:10.1136/bmjopen-2016-015776

[7] Eliacik K, Bolat N, Koçyiğit C, et al. Internet addiction, sleep and health-related life quality among obese individuals: a comparison study of the growing problems in 
Psychologische Merkmale jugendlicher Patienten vor einer Gewichtsreduktionsbehandlung adolescent health. Eat Weight Disord 2016; 21: 709-717. doi:10.1007/s40519-016$0327-\mathrm{z}$

[8] Bolton K, Kremer P, Rossthorn N, et al. The effect of gender and age on the association between weight status and health-related quality of life in Australian adolescents. BMC Public Health 2014; 14: 898. doi:10.1186/1471-2458-14-898

[9] Hestetun I, Svendsen MV, Oellingrath IM. Associations between overweight, peer problems, and mental health in 12-13-year-old Norwegian children. European Child \& Adolescent Psychiatry 2015; 24: 319-326. doi:10.1007/s00787-014-0581-4

[10] Giel KE, Zipfel S, Schweizer R, et al. Eating disorder pathology in adolescents participating in a lifestyle intervention for obesity: associations with weight change, general psychopathology and health-related quality of life. Obes Facts 2013; 6: 307-316. doi:10.1159/000354534

[11] Krause L, Ellert U, Kroll LE, et al. Gesundheitsbezogene Lebensqualität von übergewichtigen und adipösen Jugendlichen: Welche Unterschiede zeigen sich nach Sozialstatus und Schulbildung? Bundesgesundheitsblatt Gesundheitsforschung Gesundheitsschutz 2014; 57: 445-454. doi:10.1007/s00103-014-1943-2

[12] Wille N, Erhart M, Petersen C, et al. The impact of overweight and obesity on healthrelated quality of life in childhood--results from an intervention study. BMC Public Health 2008; 8: 421. doi:10.1186/1471-2458-8-421

[13] Wille N, Bullinger M, Holl R, et al. Health-related quality of life in overweight and obese youths: results of a multicenter study. Health Qual Life Outcomes 2010; 8: 36. doi:10.1186/1477-7525-8-36

[14] Ciupitu-Plath C, Wiegand S, Babitsch B. The Weight Bias Internalization Scale for Youth: Validation of a specific tool for assessing internalized weight bias among treatment-seeking German adolescents with overweight. J Pediatr Psychol 2018; 43: 4051. doi:10.1093/jpepsy/jsx079 
Psychologische Merkmale jugendlicher Patienten vor einer Gewichtsreduktionsbehandlung

364

365

366

367

368

369

370

371

372

373

374

375

376

377

378

[15] Ardelt-Gattinger E, Ring-Dimitriou S, Hofmann J, et al. Geschlechtsunterschiede bei psychologischen, ernährungs- und sportwissenschaftlichen Einflussfaktoren auf Adipositas/Übergewicht bei Kindern und Jugendlichen in Österreich. Wien Med Wochenschr 2016; 166: 111-116. doi:10.1007/s10354-015-0427-9

[16] Hughes EK, Kerr JA, Patton GC, et al. Eating disorder symptoms across the weight spectrum in Australian adolescents. Int J Eat Disord 2019; 52: 885-894. doi:10.1002/eat.23118

[17] Neumark-Sztainer D, Wall M, Story M, et al. Five-year longitudinal predictive factors for disordered eating in a population-based sample of overweight adolescents: Implications for prevention and treatment. Int J Eat Disord 2009; 42: 664-672. doi:10.1002/eat.20733

[18] Wabitsch M, Kunze D. Konsensbasierte (S2) Leitlinie zur Diagnostik, Therapie und Prävention von Übergewicht und Adipositas im Kindes- und Jugendalter. Version 15.10.2015; www.a-g-a.de

[19] Al-Khudairy L, Loveman E, Colquitt JL, et al. Diet, physical activity and behavioural interventions for the treatment of overweight or obese adolescents aged 12 to 17 years. Cochrane Database Syst Rev 2017; 6: CD012691. doi:10.1002/14651858.CD012691

[20] Harcourt BE, Pons A, Kao K-T, et al. Psychosocial measures and weight change in a clinical paediatric population with obesity. Qual Life Res 2019; 28: 1555-1564. doi:10.1007/s11136-019-02155-3

[21] Hölling H, Kurth B-M, Rothenberger A, et al. Assessing psychopathological problems of children and adolescents from 3 to 17 years in a nationwide representative sample: results of the German health interview and examination survey for children and adolescents (KiGGS). European Child \& Adolescent Psychiatry 2008; 17 Suppl 1: 3441. doi:10.1007/s00787-008-1004-1 
Psychologische Merkmale jugendlicher Patienten vor einer Gewichtsreduktionsbehandlung

[22] Ravens-Sieberer U, Ellert U, Erhart M. Gesundheitsbezogene Lebensqualität von Kindern und Jugendlichen in Deutschland. Eine Normstichprobe für Deutschland aus dem Kinder- und Jugendgesundheitssurvey (KIGGS). Bundesgesundheitsblatt Gesundheitsforschung Gesundheitsschutz 2007; 50: 810-818. doi:10.1007/s00103-0070244-4

[23] Inchley J, Currie D, Young T, Hrsg. Growing up unequal. Gender and socioeconomic differences in young people's health and well-being; Health Behaviour in School-Aged Children (HBSC) Study: international report from the 2013/2014 survey. No. 7. Health policy for children and adolescents. Copenhagen: World Health Organization, Regional Office for Europe; 2016

[24] Hilbert A. Eating Disorder Examination - Questionnaire für Kinder. Tübingen: dgvtVerlag; 2016

[25] Hilbert A, Baldofski S, Zenger M, et al. Weight bias internalization scale: psychometric properties and population norms. PLoS ONE 2014; 9: e86303. doi:10.1371/journal.pone.0086303

[26] Ravens-Sieberer U. Der Kindl-R-Fragebogen zur Erfassung der gesundheitsbezogenen Lebensqualität bei Kindern und Jugendlichen - Revidierte Form. In: Schumacher J, Klaiberg A, Brähler E, Hrsg. Diagnostische Verfahren zu Lebensqualität und Wohlbefinden. Band 2. Diagnostik für Klinik und Praxis. Göttingen, Bern, Toronto, Seattle: Hogrefe Verlag für Psychologie; 2003

[27] Woerner W, Becker A, Friedrich C, et al. Normierung und Evaluation der deutschen Elternversion des Strengths and Difficulties Questionnaire (SDQ): Ergebnisse einer repräsentativen Felderhebung. Z Kinder Jugendpsychiatr Psychother 2002; 30: 105-112. doi:10.1024//1422-4917.30.2.105

[28] Kromeyer-Hauschild K, Wabitsch M, Kunze D, et al. Perzentile für den Body-massIndex für das Kindes- und Jugendalter unter Heranziehung verschiedener deutscher 
Psychologische Merkmale jugendlicher Patienten vor einer Gewichtsreduktionsbehandlung Stichproben. Monatsschr Kinderheilkd 2001; 149: 807-818. doi:10.1007/s001120170107

[29] Steinberg L. Adolescence. 3rd ed. New York: McGraw-Hill; 1993

[30] Cohen J. Statistical power analysis for the behavioral sciences. 2. Aufl. Hillsdale, NJ: Lawrence Earlbaum Associates; 1988

[31] Kliem S, Schmidt R, Vogel M, et al. An 8-item short form of the Eating Disorder Examination-Questionnaire adapted for children (ChEDE-Q8). Int J Eat Disord 2017; 50: 679-686. doi:10.1002/eat.22658

[32] Becker A, Wang B, Kunze B, et al. Normative data of the self-report version of the German Strengths and Difficulties Questionnaire in an epidemiological setting. Zeitschrift für Kinder- und Jugendpsychiatrie und Psychotherapie 2018; 46: 523-533. doi:10.1024/1422-4917/a000589

[33] Kumar S, Kelly AS. Review of childhood obesity: From epidemiology, etiology, and comorbidities to clinical assessment and treatment. Mayo Clin Proc 2017; 92: 251-265. doi:10.1016/j.mayocp.2016.09.017

Die veröffentlichte Verlagsversion ist verfügbar unter / The final publication is available at:

434 C 2020. Thieme. All rights reserved 